Mathematical Modelling AND AnAlysis

Volume 16 Number 4, December 2011, 509-526

http://dx.doi.org/10.3846/13926292.2011.627382

(c) Vilnius Gediminas Technical University, 2011
Publisher: Taylor\&Francis and VGTU

http://www.tandfonline.com/TMMA

Online ISSN: $1648-3510$

Print ISSN: 1392-6292

\title{
On Quasi-Gasdynamic System of Equations with General Equations of State and its Application*
}

\author{
Alexander Zlotnik ${ }^{a, b}$ and Vladimir Gavrilin ${ }^{b}$ \\ ${ }^{a}$ Department of Mathematics, Faculty of Economics, Higher Economics School \\ Myasnitskaya 20, 101000 Moscow, Russia \\ ${ }^{b}$ Department of Mathematical Modelling, Moscow Power Engineering Institute \\ Krasnokazarmennaya 14, 111250 Moscow, Russia \\ E-mail(corresp.): azlotnik2008@gmail.com \\ E-mail: gavrilinva@gmail.com
}

Received June 13, 2011; revised August 12, 2011; published online November 1, 2011

\begin{abstract}
A quasi-gasdynamic system of equations with a mass force and a heat source is well known in the case of the perfect polytropic gas. In the paper, the system is extended to the case of general equations of gas state satisfying thermodynamic stability conditions. The entropy balance equation is studied. The validity of the non-negativity property is algebraically analyzed for the entropy production. Two different forms are derived for its relaxation summands. It is proved that under a condition on the heat source intensity, the non-negativity property is valid.

An application to one-dimensional Euler real gas dynamics equations is given. A two-level explicit symmetric in space finite-difference scheme is constructed. The scheme is tested in the cases of the stiffened gas and the Van der Waals gas equations of state.
\end{abstract}

Keywords: quasi-gasdynamic equations, general state equations, the entropy balance equation, Euler equations, finite-difference methods.

AMS Subject Classification: 76N15; 76M20.

\section{Introduction}

A quasi-gasdynamic (QGD) system of equations forms the basis for the construction of kinetically consistent finite-difference and finite volume methods for solving problems in gas dynamics $[2,3,7]$. Concerning the presence of heat sources, the corresponding QGD system [3] is not only important in itself, but

* This work was supported by the Russian Foundation for Basic Research (projects 09-0100600 and 10-01-90005-Bel) and the Federal Agency for Science and Innovations (state contract 14.740.11.0875). 
also in connection with solving more complicated problems of radiation gas dynamics [9].

The QGD system of equations was previously used and studied only in the case of a perfect polytropic gas (or barotropic gas), and several issues of its mathematical theory have been recently considered in $[10,11,15]$. But for a lot of applications it is obligatory to use more complicated state equations.

Consequently it is important to build such a generalization of the QGD system in the form of $[3,7]$ to general state equations satisfying the conditions of thermodynamic stability which would preserve the property of non-negativity of entropy production as a criterion for the physical correctness of the system, and in case of a perfect polytropic gas would pass to the original QGD system. The entropy balance equation is studied and criteria for non-negativity of the entropy production are algebraically analyzed, first, in the absence and then in the presence of the heat sources. Two different forms of writing the entropy production are derived once again, first, in the absence and then in the presence of the heat sources. One of these forms is an appropriate (non-trivial) generalization of that in $[3,7]$, and the other is new. Note that if the heat sources are present, there is a condition for the product of a relaxation parameter and the power of the heat sources (which generalizes the corresponding condition for the original QGD system [3]).

Related results in the absence of the heat sources are presented in $[12,13]$; in particular, an analysis of the Petrovskii parabolicity for the generalized QGD system is given there ensuring its mathematical correctness.

As an example of application of the constructed QGD system, we consider a numerical approximation for the one-dimensional Euler system of equations for real gas dynamics. A two-level explicit symmetric in space finite-difference scheme is constructed. The scheme is tested numerically in two well-known cases: (1) for the stiffened gas; (2) for the Van der Waals gas state equations. The results confirm efficiency of the scheme.

\section{Quasi-Gasdynamic System of Equations with General State Equations and Heat Sources}

The QGD system of equations in the form $[3,7]$ taking into account the mass forces and heat sources can be written as the following equations of mass, momentum and total energy balances

$$
\begin{aligned}
& \partial_{t} \rho+\operatorname{div}[\rho(\mathbf{u}-\mathbf{w})]=0, \\
& \partial_{t}(\rho \mathbf{u})+\operatorname{div}[\rho(\mathbf{u}-\mathbf{w}) \otimes \mathbf{u}]+\nabla p=\operatorname{div} \Pi+[\rho-\tau \operatorname{div}(\rho \mathbf{u})] \mathbf{F}, \\
& \partial_{t} E+\operatorname{div}[(E+p)(\mathbf{u}-\mathbf{w})]=\operatorname{div}(-\mathbf{q})+\operatorname{div}(\Pi \mathbf{u})+\rho(\mathbf{u}-\mathbf{w}) \cdot \mathbf{F}+Q .
\end{aligned}
$$

Here the viscous stress tensor $\Pi$ has the form

$$
\Pi=\Pi_{N S}+\rho \mathbf{u} \otimes \widehat{\mathbf{w}}+\tau[\mathbf{u} \nabla p+\gamma p \operatorname{div} \mathbf{u}-(\gamma-1) Q] I,
$$

where $\Pi_{N S}$ is the classical Navier-Stokes viscous stress tensor

$$
\Pi_{N S}=\mu\left[2 \mathbb{D}-\frac{2}{3}(\operatorname{div} \mathbf{u}) I\right], \quad \mathbb{D}_{i j}=\frac{1}{2}\left(\partial_{i} u_{j}+\partial_{j} u_{i}\right)
$$


with the dynamic viscosity coefficient $\mu=\mu(\rho, \theta)>0$ and zero bulk viscosity coefficient, and $I$ is the identity tensor (of order $n$ ). The heat flux $\mathbf{q}$ is given by

$$
-\mathbf{q}=\varkappa \nabla \theta+\tau\left[\rho\left(\mathbf{u} \nabla \varepsilon-\frac{p}{\rho^{2}} \mathbf{u} \nabla \rho\right)-Q\right] \mathbf{u},
$$

where $\varkappa=\varkappa(\rho, \theta)>0$ is the thermal conductivity coefficient. The auxiliary vectors $\mathbf{w}$ and $\widehat{\mathbf{w}}$ are

$$
\mathbf{w}=\frac{\tau}{\rho}[\operatorname{div}(\rho \mathbf{u} \otimes \mathbf{u})+\nabla p-\rho \mathbf{F}], \quad \widehat{\mathbf{w}}=\frac{\tau}{\rho}[\rho(\mathbf{u} \nabla) \mathbf{u}+\nabla p-\rho \mathbf{F}] .
$$

The operators div and $\nabla=\left(\partial_{1}, \ldots, \partial_{n}\right)$ are taken with respect to the spatial variables $x=\left(x_{1}, \ldots, x_{n}\right)$, where $n \geqslant 1$. Also $\partial_{i}$ and $\partial_{t}$ are the partial derivatives in $x_{i}$ and $t$. The divergence of a tensor is taken with respect to its first index. The signs $\otimes$ and $\cdot$ denote the tensor and inner products of vectors, and in the inner products such as $\mathbf{u} \cdot \nabla \varphi$ the sign $\cdot$ is omitted for brevity. Below, summation from 1 to $n$ is supposed over repeated indices $i, j$.

We consider the gas density $\rho>0$, the velocity $\mathbf{u}=\left(u_{1}, \ldots, u_{n}\right)$, the absolute temperature $\theta>0$ as the basic unknown functions. In addition, the equations include the total energy $E=\frac{1}{2} \rho|\mathbf{u}|^{2}+\rho \varepsilon$, the pressure $p$ and the specific internal energy $\varepsilon$. The relaxation parameter $\tau$ is given by $\tau=\frac{\mu}{\alpha_{S} p}$, where $\alpha_{S}>0$ is the Schmidt number. The functions $\mathbf{F}=\mathbf{F}(x, t)$ and $Q=$ $Q(x, t) \geqslant 0$ are the density of given mass forces and the power of given heat sources. In $[3,7]$, this system of equations is considered only in the case of a perfect polytropic gas, where

$$
p=(\gamma-1) \rho \varepsilon, \quad \varepsilon=c_{v} \theta, \quad \gamma=\text { const }>1, \quad c_{v}=\text { const }>0 .
$$

In this paper, we take general state equations $p=p(\rho, \theta), \varepsilon=\varepsilon(\rho, \theta)$ linked by the Maxwell relation

$$
p=\theta p_{\theta}+\rho^{2} \varepsilon_{\rho} \quad \text { in } D_{0}
$$

and satisfying the thermodynamic stability conditions of the form

$$
p_{\rho} \geqslant 0, \quad \varepsilon_{\theta}>0 \quad \text { in } D_{0},
$$

where $D_{0}$ is the range of the pair of functions $(\rho, \theta)$ in the QGD system (the case $D_{0}=(0, \infty) \times(0, \infty)$ is not excluded $)$ and, for example, $p_{\rho}$ and $p_{\theta}$ are partial derivatives of the function $p=p(\rho, \theta)$. If actually $p_{\rho}>0$ in $D_{0}$, the latter condition can be rewritten in a known equivalent form of $c_{p}>c_{v} \equiv \varepsilon_{\theta}>0$ in $D_{0}$, where $c_{p}$ and $c_{v}$ are the specific heats of gas at constant pressure and at constant volume, for example see [6]. Including of the case $p_{\rho}=0$ allows us to cover one of the simplest models for a two-phase gas/liquid mixture.

We generalize the QGD system of equations replacing term $\gamma p \operatorname{div} \mathbf{u}$ by $\rho C_{s}^{2} \operatorname{div} \mathbf{u}$ and term $(\gamma-1) Q$ by $\left(\gamma_{Q}-1\right) Q$ in the viscous stress tensor $\Pi$ :

$$
\Pi=\Pi_{N S}+\rho \mathbf{u} \otimes \widehat{\mathbf{w}}+\tau\left[\mathbf{u} \nabla p+\rho C_{s}^{2} \operatorname{div} \mathbf{u}-\left(\gamma_{Q}-1\right) Q\right] I .
$$

Here $C_{s} \geqslant 0$ is the speed of sound in the gas defined by

$$
C_{s}^{2}=p_{\rho}+\frac{\theta p_{\theta}^{2}}{\rho^{2} \varepsilon_{\theta}},
$$


and the quantity $\gamma_{Q}=\gamma_{Q}(\rho, \theta)$ is such that $\gamma_{Q}-1=\frac{p_{\theta}}{\rho \varepsilon_{\theta}}$. Derivation of the latter formula is given below; it turns out that under the above generalization of the term $\gamma p \operatorname{div} \mathbf{u}$, the form of the coefficient $\gamma_{Q}-1$ is uniquely determined.

By virtue of the stability conditions (2.5), formula (2.6) is correct (in particular, it is ensured that $\left.C_{s}^{2} \geqslant 0\right)$. Moreover, the equality

$$
C_{s}^{2}=\frac{c_{p}}{c_{v}} p_{\rho}
$$

holds [6, p. 155] and therefore $\rho C_{s}^{2}=\frac{c_{p}}{c_{v}} \rho p_{\rho}$. Notice that formula (2.6) follows easily from (2.7) and

$$
c_{p}-c_{v}=\theta p_{\theta}^{2} /\left(\rho^{2} p_{\rho}\right) \text {, }
$$

see $[6$, p. 47].

In the particular case of the perfect polytropic gas we get $\rho p_{\rho}=p, \theta p_{\theta}=p$, $\theta \varepsilon_{\theta}=\varepsilon$, and therefore $C_{s}^{2}=\gamma(\gamma-1) \varepsilon, \rho C_{s}^{2}=\gamma p$ and $\gamma_{Q}-1=\gamma-1$; also $\frac{c_{p}}{c_{v}}=\gamma$. Let $\Gamma=\frac{\rho p_{\rho}}{p}$. In another (barotropic) special case, where $p=p(\rho)$, we have $C_{s}^{2}=p_{\rho}$ and $\rho C_{s}^{2}=\Gamma p$ as in [11].

Following [11], below we also consider the more generic Navier-Stokes viscous stress tensor

$$
\Pi_{N S}=\mu\left[2 \mathbb{D}-\frac{2}{3}(\operatorname{div} \mathbf{u}) I\right]+\lambda(\operatorname{div} \mathbf{u}) I
$$

with the bulk viscosity coefficient $\lambda=\lambda(\rho, \theta) \geqslant 0$ and, like [10, 15], we take an arbitrary relaxation parameter $\tau=\tau(\rho, \theta)>0$.

We introduce the entropy $s=s(\rho, \varepsilon)$ by the Gibbs formulas

$$
s_{\rho}=-\frac{p}{\rho^{2} \theta}, \quad s_{\varepsilon}=\frac{1}{\theta} .
$$

Since then

$$
\theta \nabla s=\nabla \varepsilon-\frac{p}{\rho^{2}} \nabla \rho,
$$

the heat flux can be expressed using a shorter formula

$$
-\mathbf{q}=\varkappa \nabla \theta+\tau(\rho \theta \mathbf{u} \nabla s-Q) \mathbf{u}
$$

We introduce the total time derivative

$$
D_{t} \varphi:=\partial_{t}(\rho \varphi)+\operatorname{div}[\rho \varphi(\mathbf{u}-\mathbf{w})]=\rho \partial_{t} \varphi+\rho(\mathbf{u}-\mathbf{w}) \nabla \varphi,
$$

see (2.1). We subtract the total energy equation (2.3) from the momentum equation (2.2) multiplied scalarly by $\mathbf{u}$, take into account formulas

$$
\begin{aligned}
\partial_{t}(\rho \mathbf{u}) \cdot \mathbf{u}+\operatorname{div}[\rho(\mathbf{u}-\mathbf{w}) \otimes \mathbf{u}] \cdot \mathbf{u} & =\partial_{t}\left(\frac{1}{2} \rho|\mathbf{u}|^{2}\right)+\operatorname{div}\left[\frac{1}{2} \rho|\mathbf{u}|^{2}(\mathbf{u}-\mathbf{w})\right] \\
\mathbf{w}=\widehat{\mathbf{w}}+\frac{\tau}{\rho} \operatorname{div}(\rho \mathbf{u}) \mathbf{u} & =\widehat{\mathbf{w}}+\frac{\tau}{\rho}(\mathbf{u} \nabla \rho+\rho \operatorname{div} \mathbf{u}) \mathbf{u}
\end{aligned}
$$

and thus derive the equation of specific internal energy balance

$$
D_{t} \varepsilon=\operatorname{div}(-\mathbf{q})+\Pi_{i j} \partial_{i} u_{j}-p \operatorname{div}(\mathbf{u}-\mathbf{w})+\mathbf{w} \nabla p-\rho \widehat{\mathbf{w}} \cdot \mathbf{F}+Q .
$$


Here the term $\Pi_{i j} \partial_{i} u_{j}$ has the form

$$
\begin{aligned}
\Pi_{i j} \partial_{i} u_{j}= & 2 \mu \mathbb{D}_{i j} \mathbb{D}_{i j}+\left(\lambda-\frac{2}{3} \mu\right)(\operatorname{div} \mathbf{u})^{2} \\
& +\tau\left\{\rho(\mathbf{u} \nabla) \mathbf{u} \cdot \widehat{\mathbf{w}}+\left[\mathbf{u} \nabla p+\rho C_{s}^{2} \operatorname{div} \mathbf{u}-\left(\gamma_{Q}-1\right) Q\right] \operatorname{div} \mathbf{u}\right\} .
\end{aligned}
$$

Theorem 1. For the above generalized QGD system, the equation of entropy balance

$$
D_{t} s=\operatorname{div}(-\mathbf{q} / \theta)+\Xi / \theta
$$

holds, where the entropy production $\frac{1}{\theta} \Xi$ is expressed by formulas

$$
\begin{gathered}
\Xi=\Xi_{N S, 0}+\frac{\rho}{\tau}|\widehat{\mathbf{w}}|^{2}+\frac{\tau p_{\rho}}{\rho}[\operatorname{div}(\rho \mathbf{u})]^{2} \\
+\frac{\tau \rho \varepsilon_{\theta}}{\theta}\left(\frac{\theta p_{\theta}}{\rho \varepsilon_{\theta}} \operatorname{div} \mathbf{u}+\mathbf{u} \nabla \theta-\frac{Q}{2 \rho \varepsilon_{\theta}}\right)^{2}+Q\left(1-\frac{\tau Q}{4 \rho \theta \varepsilon_{\theta}}\right), \\
\Xi_{N S, 0}=2 \mu \mathbb{D}_{i j} \mathbb{D}_{i j}+\left(\lambda-\frac{2}{3} \mu\right)(\operatorname{div} \mathbf{u})^{2}+\frac{\varkappa}{\theta}|\nabla \theta|^{2} \geqslant 0 \text { for } n=1,2,3 .
\end{gathered}
$$

The entropy production can be expressed also by formulas

$$
\begin{aligned}
\Xi= & \Xi_{N S, 0}+\frac{\rho}{\tau}|\widehat{\mathbf{w}}|^{2}+\frac{\tau}{\rho C_{s}^{2}}\left(\rho C_{s}^{2} \operatorname{div} \mathbf{u}+\mathbf{u} \nabla p-\frac{\left(\gamma_{Q}-1\right) Q}{2}\right)^{2} \\
& +\frac{\tau \rho \theta}{c_{p}}\left(\mathbf{u} \nabla s-\frac{Q}{2 \rho \theta}\right)^{2}+Q\left(1-\frac{\tau Q}{4 \rho \theta \varepsilon_{\theta}}\right)
\end{aligned}
$$

and (2.15) provided that $p_{\rho}>0$. Under the condition

$$
\frac{\tau Q}{4 \rho \theta \varepsilon_{\theta}} \leqslant 1
$$

the entropy production is non-negative: $\frac{1}{\theta} \Xi \geqslant 0$ for $n=1,2,3$.

Proof. The proof comprises several steps.

1. In steps $1-3$, in the viscous stress tensor $\Pi$ we consider the term $\gamma_{p} p \operatorname{div} \mathbf{u}$ with the coefficient $\gamma_{p}=\gamma_{p}(\rho, \theta)$ of general form instead of $\rho C_{s}^{2}$ div $\mathbf{u}$. First, in a standard manner we write that

$$
D_{t} s=\frac{1}{\theta}\left(p D_{t} \frac{1}{\rho}+D_{t} \varepsilon\right)=\frac{1}{\theta}\left[p \operatorname{div}(\mathbf{u}-\mathbf{w})+D_{t} \varepsilon\right]=\operatorname{div}\left(-\frac{\mathbf{q}}{\theta}\right)+\frac{1}{\theta} \Xi
$$

with the help of the Gibbs formulas (2.8) and the internal energy balance equation (2.12), where

$$
\Xi=\frac{1}{\theta}(-\mathbf{q}) \nabla \theta+\Pi_{i j} \partial_{i} u_{j}+\mathbf{w} \nabla p-\rho \widehat{\mathbf{w}} \cdot \mathbf{F}+Q
$$

We use formulas (2.13) and (2.10). Since

$$
(\rho \mathbf{u} \nabla) \mathbf{u} \cdot \widehat{\mathbf{w}}+\mathbf{w} \nabla p-\rho \widehat{\mathbf{w}} \cdot \mathbf{F}=\frac{\rho}{\tau}|\widehat{\mathbf{w}}|^{2}+\tau\left(\frac{1}{\rho} \mathbf{u} \nabla \rho+\operatorname{div} \mathbf{u}\right) \mathbf{u} \nabla p
$$


by taking into account formula $(2.11)$, by rearrangement the terms we get

$$
\Xi=\Xi_{N S, 0}+\frac{\rho}{\tau}|\widehat{\mathbf{w}}|^{2}+Q+\tau \Xi^{\prime}
$$

where

$$
\begin{aligned}
\Xi_{N S, 0}= & 2 \mu \mathbb{D}_{i j} \mathbb{D}_{i j}+\left(\lambda-\frac{2}{3} \mu\right)(\operatorname{div} \mathbf{u})^{2}+\frac{\varkappa}{\theta}|\nabla \theta|^{2} \\
\Xi^{\prime}= & \gamma_{p} p(\operatorname{div} \mathbf{u})^{2}+2(\operatorname{div} \mathbf{u}) \mathbf{u} \nabla p+\frac{1}{\rho}(\mathbf{u} \nabla \rho) \mathbf{u} \nabla p+\rho(\mathbf{u} \nabla s) \mathbf{u} \nabla \theta \\
& -Q\left[\left(\gamma_{Q}-1\right) \operatorname{div} \mathbf{u}+\frac{1}{\theta} \mathbf{u} \nabla \theta\right] .
\end{aligned}
$$

Note that $\frac{1}{\theta} \Xi_{N S, 0}$ is the entropy production for the Navier-Stokes compressible gas equations for $Q=0$. Inequality (2.15) follows from inequalities $\mathbb{D}_{i j} \mathbb{D}_{i j} \geqslant \mathbb{D}_{i i} \mathbb{D}_{i i} \geqslant \frac{1}{n}(\operatorname{div} \mathbf{u})^{2}$.

2. The rest of the proof is devoted to the analysis of term $\Xi^{\prime}$. Let first $Q=0$. Using formula (2.9) and substituting $\nabla p=p_{\rho} \nabla \rho+p_{\theta} \nabla \theta$ and $\nabla \varepsilon=$ $\varepsilon_{\rho} \nabla \rho+\varepsilon_{\theta} \nabla \theta$, from (2.18) we obtain

$$
\Xi^{\prime}=\gamma_{p} p(\operatorname{div} \mathbf{u})^{2}+2(\operatorname{div} \mathbf{u})\left(p_{\rho} \mathbf{u} \nabla \rho+p_{\theta} \mathbf{u} \nabla \theta\right)+\frac{p_{\rho}}{\rho}(\mathbf{u} \nabla \rho)^{2}+\frac{\rho \varepsilon_{\theta}}{\theta}(\mathbf{u} \nabla \theta)^{2}
$$

here we take into account that

$$
\left[\frac{p_{\theta}}{\rho}+\frac{\rho}{\theta}\left(\varepsilon_{\rho}-\frac{p}{\rho^{2}}\right)\right](\mathbf{u} \nabla \rho) \mathbf{u} \nabla \theta=0
$$

by virtue of the Maxwell relation (2.4). Thus $\Xi^{\prime}=G y \cdot y$, where

$$
G:=\left(\begin{array}{ccc}
\gamma_{p} p & p_{\rho} & p_{\theta} \\
p_{\rho} & \frac{p_{\rho}}{\rho} & 0 \\
p_{\theta} & 0 & \frac{\rho \varepsilon_{\theta}}{\theta}
\end{array}\right), \quad y:=\left(\begin{array}{c}
\operatorname{div} \mathbf{u} \\
\mathbf{u} \nabla \rho \\
\mathbf{u} \nabla \theta
\end{array}\right)
$$

is a quadratic form of three variables.

The quadratic form $G y \cdot y$ is positive semidefinite if and only if all its principal minors are nonnegative [5]. In particular, regardless of the previous study inequalities

$$
\gamma_{p} p \geqslant 0, \quad p_{\rho} \geqslant 0, \quad \varepsilon_{\theta} \geqslant 0
$$

have to be satisfied, compare with the thermodynamic stability conditions (2.5). Note also that

$$
\operatorname{det} G=\frac{p_{\rho} \varepsilon_{\theta}}{\theta}\left(\gamma_{p} p-\rho C_{s}^{2}\right)
$$

By virtue of (2.5) it is easy to see that the condition on the minors is reduced to a unique one $\gamma_{p} p \geqslant \rho C_{s}^{2}$. So under conditions (2.5), the latter condition and for $Q=0$, the entropy production is nonnegative (for $n=1,2,3$ ).

3. The quadratic form $\Xi^{\prime}=G y \cdot y$ can be explicitly represented as the sum of squares in several ways. To generalize the representation from $[3,7]$, we 
transform expression (2.19) as follows:

$$
\begin{aligned}
\Xi^{\prime} & =\gamma_{p} p(\operatorname{div} \mathbf{u})^{2}+\frac{p_{\rho}}{\rho}\left[(\rho \operatorname{div} \mathbf{u}+\mathbf{u} \nabla \rho)^{2}-\rho^{2}(\operatorname{div} \mathbf{u})^{2}\right] \\
& +\frac{\rho}{\varepsilon_{\theta} \theta}\left[\left(\frac{\theta p_{\theta}}{\rho} \operatorname{div} \mathbf{u}+\varepsilon_{\theta} \mathbf{u} \nabla \theta\right)^{2}-\frac{\theta^{2} p_{\theta}^{2}}{\rho^{2}}(\operatorname{div} \mathbf{u})^{2}\right] \\
& =\left(\gamma_{p} p-\rho C_{s}^{2}\right)(\operatorname{div} \mathbf{u})^{2}+\frac{p_{\rho}}{\rho}[\operatorname{div}(\rho \mathbf{u})]^{2}+\frac{\rho}{\theta \varepsilon_{\theta}}\left(\frac{\theta p_{\theta}}{\rho} \operatorname{div} \mathbf{u}+\varepsilon_{\theta} \mathbf{u} \nabla \theta\right)^{2} .
\end{aligned}
$$

In the particular case of the perfect polytropic gas and for $\gamma_{p}=\gamma$, the latter expression takes the familiar form $[3,7]$

$$
\Xi^{\prime}=\frac{p}{\rho^{2}}[\operatorname{div}(\rho \mathbf{u})]^{2}+\frac{\rho}{\varepsilon}\left(\frac{p}{\rho} \operatorname{div} \mathbf{u}+\mathbf{u} \nabla \varepsilon\right)^{2}
$$

On the other hand, expression (2.18) in the case $Q=0$ can be transformed alternatively

$$
\begin{gathered}
\Xi^{\prime}=\left(\gamma_{p} p-\rho C_{s}^{2}\right)(\operatorname{div} \mathbf{u})^{2}+\frac{1}{\rho C_{s}^{2}}\left(\rho C_{s}^{2} \operatorname{div} \mathbf{u}+\mathbf{u} \nabla p\right)^{2} \\
-\frac{1}{\rho C_{s}^{2}}(\mathbf{u} \nabla p)^{2}+\frac{1}{\rho}(\mathbf{u} \nabla \rho) \mathbf{u} \nabla p+\rho(\mathbf{u} \nabla \theta) \mathbf{u} \nabla s
\end{gathered}
$$

for $p_{\rho}>0$. Taking into account formula (2.9) for $\nabla s$ and the Maxwell relation (2.4), we have the following system of equations to find $\nabla \rho$ and $\nabla \theta$ through $\nabla p$ and $\theta \nabla s$ :

$$
p_{\rho} \nabla \rho+p_{\theta} \nabla \theta=\nabla p, \quad-\frac{\theta p_{\theta}}{\rho^{2}} \nabla \rho+\varepsilon_{\theta} \nabla \theta=\theta \nabla s .
$$

Its determinant is equal to $\varepsilon_{\theta} C_{s}^{2}>0$. The solution of the system of equations is as follows

$$
\nabla \rho=\frac{1}{\varepsilon_{\theta} C_{s}^{2}}\left(\varepsilon_{\theta} \nabla p-\theta p_{\theta} \nabla s\right), \quad \nabla \theta=\frac{1}{\varepsilon_{\theta} C_{s}^{2}}\left(\frac{\theta p_{\theta}}{\rho^{2}} \nabla p+\theta p_{\rho} \nabla s\right) .
$$

Hence, by virtue of relation (2.7) for $C_{s}^{2}$, we obtain

$$
-\frac{1}{\rho C_{s}^{2}}(\mathbf{u} \nabla p)^{2}+\frac{1}{\rho}(\mathbf{u} \nabla \rho) \mathbf{u} \nabla p+\rho(\mathbf{u} \nabla \theta) \mathbf{u} \nabla s=\frac{\rho \theta p_{\rho}}{\varepsilon_{\theta} C_{s}^{2}}(\mathbf{u} \nabla s)^{2}=\frac{\rho \theta}{c_{p}}(\mathbf{u} \nabla s)^{2} .
$$

Thus we have derived a representation of another type [12]

$$
\Xi^{\prime}=\left(\gamma_{p} p-\rho C_{s}^{2}\right)(\operatorname{div} \mathbf{u})^{2}+\frac{1}{\rho C_{s}^{2}}\left(\rho C_{s}^{2} \operatorname{div} \mathbf{u}+\mathbf{u} \nabla p\right)^{2}+\frac{\rho \theta}{c_{p}}(\mathbf{u} \nabla s)^{2} .
$$
form

4. Now let $\gamma_{p} p=\rho C_{s}^{2}$ and $Q>0$. We rewrite the expression for $\Xi^{\prime}$ in the

$$
\Xi^{\prime}=G y \cdot y-Q b \cdot y=: J(y)
$$


with the vector $b:=\left(\gamma_{Q}-1,0,1 / \theta\right)$. Since the matrix $G \geqslant 0$ is singular, the function $J$ is bounded from below on $\mathbb{R}^{3}$ if and only if $b \perp \operatorname{Ker} G$ or, equivalently, $b \in \operatorname{Im} G$.

Let $G^{(1)}, G^{(2)}, G^{(3)}$ be the successive columns of $G$. Let $p_{\rho}>0$. Then $\operatorname{rank} G=2$, and $\operatorname{Im} G$ is the linear span, for example, of $G^{(2)}$ and $G^{(3)}$. Since $b_{2}=G_{2}^{(3)}=0$, actually $b \in \operatorname{Im} G$ is collinear $G^{(3)}$, i.e. $b=\alpha G^{(3)}$. If, however, $p_{\rho}=0$, then $\operatorname{rank} G=1$, and $\operatorname{Im} G$ is the linear span of $G^{(3)}$, and once again $b=\alpha G^{(3)}$. Thus $\alpha=\frac{1}{\rho \varepsilon_{\theta}}$, and $\gamma_{Q}-1$ is uniquely determined:

$$
\gamma_{Q}-1=\alpha p_{\theta}=\frac{p_{\theta}}{\rho \varepsilon_{\theta}} .
$$

Below, this choice is assumed to be done.

Let $y_{*}$ be any solution of a system of equations $G y=\frac{Q}{2} b$. Then

$$
J_{*}:=\min _{\mathbb{R}^{3}} J(y)=-G y_{*} \cdot y_{*}=-\frac{Q}{2} b \cdot y_{*} .
$$

Since $b=\alpha G^{(3)}$, we can take $y_{*}=(0,0, Q \alpha / 2)$, and therefore

$$
J_{*}=-\frac{Q^{2}}{4} \frac{\alpha}{\theta}=-\frac{Q^{2}}{4 \rho \theta \varepsilon_{\theta}} .
$$

Consequently

$$
Q+\tau \Xi^{\prime} \geqslant Q\left(1-\frac{\tau Q}{4 \rho \theta \varepsilon_{\theta}}\right)
$$

Obviously, the last quantity and therefore the entropy production are nonnegative (for $n=1,2,3$ ) provided that $\tau Q \leq 4 \rho \theta \varepsilon_{\theta}$.

For $p_{\theta}>0$, this condition can also be rewritten as

$$
\tau\left(\gamma_{Q}-1\right) Q \leqslant 4 \theta p_{\theta} .
$$

In the particular case of the perfect polytropic gas it takes the simplest form

$$
\tau(\gamma-1) Q \leqslant 4 p
$$

5. It is easy to generalize the representation (2.20) to the case $Q \geqslant 0$. Since

$$
\left(\gamma_{Q}-1\right) \operatorname{div} \mathbf{u}+\frac{1}{\theta} \mathbf{u} \nabla \theta=\frac{1}{\theta}\left(\frac{\theta p_{\theta}}{\rho \varepsilon_{\theta}} \operatorname{div} \mathbf{u}+\mathbf{u} \nabla \theta\right),
$$

for $\gamma_{p} p=\rho C_{s}^{2}$ we have

$$
\Xi^{\prime}=\frac{p_{\rho}}{\rho}[\operatorname{div}(\rho \mathbf{u})]^{2}+\frac{\rho \varepsilon_{\theta}}{\theta}\left(\frac{\theta p_{\theta}}{\rho \varepsilon_{\theta}} \operatorname{div} \mathbf{u}+\mathbf{u} \nabla \theta-\frac{Q}{2 \rho \varepsilon_{\theta}}\right)^{2}-\frac{Q^{2}}{4 \rho \theta \varepsilon_{\theta}} .
$$

Thus representation (2.14) is valid.

We generalize representation (2.22) as well, for $\gamma_{p} p=\rho C_{s}^{2}$ and $p_{\rho}>0$. Let us seek it in the form

$$
\begin{aligned}
\Xi^{\prime}= & \frac{1}{\rho C_{s}^{2}}\left(\rho C_{s}^{2} \operatorname{div} \mathbf{u}+\mathbf{u} \nabla p-\frac{Q}{2} c\right)^{2}+\frac{\rho \theta}{c_{p}}\left(\mathbf{u} \nabla s-\frac{Q}{2} d\right)^{2} \\
& -\frac{Q^{2}}{4}\left(\frac{c^{2}}{\rho C_{s}^{2}}+\frac{\rho \theta d^{2}}{c_{p}}\right) .
\end{aligned}
$$


The coefficients $c=c(\rho, \theta)$ and $d=d(\rho, \theta)$ have to satisfy the equation

$$
\left(\operatorname{div} \mathbf{u}+\frac{1}{\rho C_{s}^{2}} \mathbf{u} \nabla p\right) c+\frac{\rho \theta}{c_{p}}(\mathbf{u} \nabla s) d=\left(\gamma_{Q}-1\right) \operatorname{div} \mathbf{u}+\frac{1}{\theta} \mathbf{u} \nabla \theta
$$

Therefore, we have to set $c=\gamma_{Q}-1$, and then by formulas (2.21) and (2.7) an equation

$$
\frac{c}{\rho C_{s}^{2}}\left(p_{\rho} \mathbf{u} \nabla \rho+p_{\theta} \mathbf{u} \nabla \theta\right)+\frac{\rho p_{\rho} d}{\varepsilon_{\theta} C_{s}^{2}}\left(-\frac{\theta p_{\theta}}{\rho^{2}} \mathbf{u} \nabla \rho+\varepsilon_{\theta} \mathbf{u} \nabla \theta\right)=\frac{1}{\theta} \mathbf{u} \nabla \theta
$$

has to be valid. This occurs if $c$ and $d$ satisfy a system of equations

$$
c-\frac{\theta p_{\theta}}{\varepsilon_{\theta}} d=0, \quad \frac{p_{\theta}}{\rho} c+\rho p_{\rho} d=\frac{C_{s}^{2}}{\theta} .
$$

Its determinant is equal to $\rho C_{s}^{2}>0$, see (2.6). Hence

$$
c=\gamma_{Q}-1=\frac{p_{\theta}}{\rho \varepsilon_{\theta}}, \quad d=\frac{1}{\rho \theta} .
$$

Formula (2.23) is obtained once again. In addition, we have

$$
\frac{c^{2}}{\rho C_{s}^{2}}+\frac{\rho \theta d^{2}}{c_{p}}=\frac{1}{\rho \theta \varepsilon_{\theta}}\left(\frac{\theta p_{\theta}^{2}}{\rho^{2} \varepsilon_{\theta} C_{s}^{2}}+\frac{p_{\rho}}{C_{s}^{2}}\right)=\frac{1}{\rho \theta \varepsilon_{\theta}},
$$

which eventually leads from formula (2.24) to representation (2.16). The theorem is completely proved.

Note that if the coefficient $\frac{\tau \rho \theta}{c_{p}}$ is rewritten as $\frac{\tau \rho \theta p_{\rho}}{\varepsilon_{\theta} C_{s}^{2}}$ in formula (2.16), it remains valid under conditions $p_{\rho} \geqslant 0$ and $C_{s}^{2} \neq 0$.

Remark 1. Since

$$
\partial_{t} p=\left(p_{\rho}-\frac{p_{\theta} \varepsilon_{\rho}}{\varepsilon_{\theta}}\right) \partial_{t} \rho+\frac{p_{\theta}}{\varepsilon_{\theta}} \partial_{t} \varepsilon
$$

solutions of the classical Euler equations with general state equations and heat source satisfy

$$
\begin{aligned}
\partial_{t} p & =-\left(p_{\rho}-\frac{p_{\theta} \varepsilon_{\rho}}{\varepsilon_{\theta}}\right)(\mathbf{u} \nabla \rho+\rho \operatorname{div} \mathbf{u})-\frac{p_{\theta}}{\varepsilon_{\theta}}\left(\mathbf{u} \nabla \varepsilon+\frac{p}{\rho} \operatorname{div} \mathbf{u}-\frac{Q}{\rho}\right) \\
& =-\mathbf{u} \nabla p-\left[\rho p_{\rho}+\frac{p_{\theta}}{\rho \varepsilon_{\theta}}\left(p-\rho^{2} \varepsilon_{\rho}\right)\right] \operatorname{div} \mathbf{u}+\frac{p_{\theta}}{\rho \varepsilon_{\theta}} Q
\end{aligned}
$$

i.e. taking into account the Maxwell relation, an equation

$$
\partial_{t} p+\mathbf{u} \nabla p+\rho C_{s}^{2} \operatorname{div} \mathbf{u}=\frac{p_{\theta}}{\rho \varepsilon_{\theta}} Q
$$

holds. Therefore, the physical derivation of the QGD system of equations with a heat source (given in Section 3.6 in [3] in the case of the perfect polytropic gas) leads exactly to the presented above equations in the case of general state equations. Concerning the results of this section, see also [14]. 


\section{Application for Solving One-Dimensional Euler Real Gas Equations}

In the one-dimensional case and in the absence of the mass forces and the heat sources, for $\lambda=0$, the QGD system is simplified and can be rewritten in the form

$$
\begin{aligned}
& \partial_{t} \rho+\partial_{x} j=0, \\
& \partial_{t}(\rho u)+\partial_{x}(j u+p)=\partial_{x} \Pi, \\
& \partial_{t} E+\partial_{x} \frac{j(E+p)}{\rho}=-\partial_{x} q+\partial_{x}(\Pi u)
\end{aligned}
$$

with the scalar velocity $u$ and quantities

$$
\begin{aligned}
j & :=\rho(u-w), \quad w=\frac{\tau}{\rho} \partial_{x}\left(\rho u^{2}+p\right), \\
\Pi & =\frac{4}{3} \mu \partial_{x} u+\tau\left(\rho u^{2} \partial_{x} u+2 u \partial_{x} p+\rho C_{s}^{2} \partial_{x} u\right), \\
-q & =\varkappa \partial_{x} \theta+\tau \rho u^{2}\left(\partial_{x} \varepsilon-\frac{p}{\rho^{2}} \partial_{x} \rho\right)
\end{aligned}
$$

where $j$ is the mass flux density. Recall also that $E=\frac{1}{2} \rho u^{2}+\rho \varepsilon$.

It is convenient to exploit the state equations also in an alternative form

$$
p=P(\rho, \varepsilon), \quad \theta=\Theta(\rho, \varepsilon) .
$$

Note that then the formula for the speed of sound takes the form

$$
C_{s}^{2}=P_{\rho}+\frac{p}{\rho^{2}} P_{\varepsilon}
$$

The Euler system of equations for inviscid and non-heat-conducting gas flow is similar but contains no dissipative summands, i.e. those with the coefficients $\tau, \mu$ and $\varkappa$. To approximate it numerically, the dissipative summands are considered as artificial regularizers. Following [3, p. 64] and [7, p. 345], we connect them by formulas

$$
\mu=\alpha_{S} \tau \rho p_{\rho}(\rho, \theta), \quad \varkappa=\frac{\mu c_{p}}{\alpha_{P}}=\frac{\alpha_{S}}{\alpha_{P}} \tau \rho c_{v} C_{s}^{2},
$$

see equality (2.7) as well. Here $\alpha_{S}$ and $\alpha_{P}$ are Schmidt's and Prandtl's numbers (positive constants). The relaxation coefficient $\tau$ is chosen below related to a space mesh step and $C_{s}$.

We construct an explicit two-level in time and symmetric in space finitedifference scheme. We use a uniform mesh in space with nodes $x_{i}=(i-1) h$, $1 \leqslant i \leqslant N$, and the step $h=X /(N-1)$, where the main unknowns $\rho, u$ and $\varepsilon$ together with $p$ and $\theta$ are defined. We also use an auxiliary mesh with nodes $x_{i-1 / 2}=(i-0.5) h, 1 \leqslant i \leqslant N-1$, where the auxiliary unknowns $j, w, \Pi$ and $q$ are defined. We define the symmetric mesh averaging and central finite differences (involving both the main and auxiliary meshes)

$$
v_{a, i-1 / 2}=\frac{v_{i-1}+v_{i}}{2}, \quad \delta v_{i-1 / 2}=\frac{v_{i}-v_{i-1}}{h}, \quad \delta w_{i}=\frac{w_{i+1 / 2}-w_{i-1 / 2}}{h} .
$$


Required values of $\rho, u$ and $\varepsilon$ together with $p$ on the auxiliary mesh are calculated using the averaging $(\cdot)_{a}$.

We also use a nonuniform mesh in time with nodes $0=t_{0}<\cdots<t_{M}=t_{\text {fin }}$ and variable steps $\Delta t_{m}=t_{m+1}-t_{m}$.

Following [4], we approximate the QGD system (3.1)-(3.3) by a two-level explicit symmetric in space finite-difference scheme applying the forward finite differences in $t$ and the central finite differences in $x$ :

$$
\begin{aligned}
& \frac{\hat{\rho}-\rho}{\Delta t}+\delta j=0, \\
& \frac{\widehat{\rho u}-\rho u}{\Delta t}+\delta\left(j u_{a}+p_{a}\right)=\delta \Pi, \\
& \frac{\hat{E}-E}{\Delta t}+\delta\left(j \frac{E_{a}+p_{a}}{\rho_{a}}\right)=\delta\left(-q+\Pi u_{a}\right) .
\end{aligned}
$$

In this section, quantities marked by the hat $\hat{\cdot}$ are related to the upper time level. After calculating the values $\hat{\rho}, \widehat{\rho u}$ and $\hat{E}$ on the upper time level, we set

$$
\hat{u}=\frac{\widehat{\rho u}}{\hat{\rho}}, \quad \hat{\varepsilon}=\frac{\hat{E}}{\hat{\rho}}-\frac{1}{2} \hat{u}^{2}
$$

assuming that $\hat{\rho}>0$. We also approximate relations (3.4)-(3.6) as follows

$$
\begin{aligned}
j & =\rho_{a}\left(u_{a}-w\right), \quad w=\frac{\tau_{a}}{\rho_{a}} \delta\left(\rho u^{2}+p\right), \\
\Pi & =\frac{4}{3} \mu_{a} \delta u+\tau_{a}\left(\rho_{a} u_{a}^{2} \delta u+2 u_{a} \delta p+\rho_{a}\left(C_{s}^{2}\right)_{a} \delta u\right), \\
-q & =\varkappa_{a} \delta \theta+\tau_{a} \rho_{a} u_{a}\left(\delta \varepsilon-\frac{p_{a}}{\rho_{a}^{2}} \delta \rho\right) .
\end{aligned}
$$

For solving the Euler equations, $\tau$ is considered as a regularizing parameter defined by $\tau=\alpha h / C_{s}$. Keeping in mind the Courant stability condition, we also take the current time step in the form

$$
\Delta t=\beta \min _{1 \leqslant i \leqslant N} \frac{h}{\left|u_{i}\right|+C_{s, i}} .
$$

Here $0<\alpha<1$ and $0<\beta<1$ are fitting parameters.

We apply the scheme for two particular non-perfect polytropic gas models (the case of the perfect polytropic gas was carefully tested in [4]).

1. The rather simple stiffened gas (or two-term) model appears in a lot of papers and is described by

$$
P(\rho, \varepsilon)=(\gamma-1) \rho \varepsilon+B\left(\rho / \rho_{*}-1\right),
$$

where $\gamma>1, B$ and $\rho_{*}$ are positive parameters. Then formula (3.8) implies

$$
C_{s}^{2}=\frac{\gamma p+B}{\rho}=\gamma(\gamma-1) \varepsilon+\frac{\gamma B}{\rho_{*}}+\frac{(\gamma-1) B}{\rho}>0 .
$$


In contrast to many other schemes, in addition to (3.10) we also need the second state equation (3.7). To derive it, we assume that $\varepsilon_{\theta}=c_{v} \equiv$ const $>0$ and thus get $\varepsilon(\rho, \theta)=c_{v} \theta+\varepsilon_{0}(\rho)$. Therefore

$$
p(\rho, \theta)=(\gamma-1) \rho\left[c_{v} \theta+\varepsilon_{0}(\rho)\right]+B\left(\rho / \rho_{*}-1\right),
$$

and the Maxwell relation (2.4) implies an ordinary differential equation to find $\varepsilon_{0}(\rho)$ :

$$
\rho^{2} \varepsilon_{0}^{\prime}(\rho)=(\gamma-1) \rho \varepsilon_{0}(\rho)+B\left(\rho / \rho_{*}-1\right) .
$$

Its general solution is

$$
\varepsilon_{0}(\rho)=c_{0} \rho^{\gamma-1}+\frac{B}{\gamma \rho}-\frac{B}{(\gamma-1) \rho_{*}}
$$

with any constant $c_{0}$. To ensure the property $\varepsilon_{0}(\rho) \geqslant 0$ for any $\rho>0$, we consider $c_{0}>0$. Then the minimum point of $\varepsilon_{0}(\rho)$ is

$$
\rho_{\min }=\left[\frac{B}{\gamma(\gamma-1) c_{0}}\right]^{1 / \gamma}
$$

and therefore

$$
\min _{\rho>0} \varepsilon_{0}(\rho)=\varepsilon_{0}\left(\rho_{\min }\right)=\frac{B}{(\gamma-1)}\left(\frac{1}{\rho_{\min }}-\frac{1}{\rho_{*}}\right) .
$$

Consequently $c_{0}$ has to satisfy an inequality

$$
c_{0} \geqslant \frac{B}{\gamma(\gamma-1) \rho_{*}^{\gamma}},
$$

and finally we choose $c_{0}=B /\left(\gamma(\gamma-1) \rho_{*}^{\gamma}\right)$.

Formula (3.11) implies

$$
p_{\rho}(\rho, \theta)=(\gamma-1) c_{v} \theta+c_{0} \gamma(\gamma-1) \rho^{\gamma-1}
$$

so the thermodynamic stability conditions (2.5) are valid for any $\theta>0$ and $\rho>0$ provided that $c_{v}>0$ and $c_{0} \geqslant 0$.

Note that since the coefficient $\varkappa$ contains the multiplier $c_{v}$, see $(3.9)$, for $c_{v}=$ const, hereafter the function $c_{v} \theta$ can be easily excluded from the system of equations.

For the stiffened gas model, we exploit three tests from [8] with different gas flow configurations. Let $B=\rho_{*}=1, \gamma=1.4$ and $X=1$. In test 1 , initial data for $\rho, u$ and $p$ are given by

$$
\rho_{0}(x)=\left\{\begin{array}{ll}
1 & \text { for } x \leqslant 0.5, \\
0.125 & \text { for } x>0.5,
\end{array} \quad u_{0}(x)=0, \quad p_{0}(x)= \begin{cases}8 & \text { for } x \leqslant 0.5 \\
0.1 & \text { for } x>0.5\end{cases}\right.
$$

and $t_{\text {fin }}=0.075$. Next, in test 2 , the initial data are given by

$$
\rho_{0}(x)=\left\{\begin{array}{ll}
0.8 & \text { for } x \leqslant 0.5, \\
1 & \text { for } x>0.5,
\end{array} \quad u_{0}(x)=0, \quad p_{0}(x)= \begin{cases}10 & \text { for } x \leqslant 0.5 \\
0.1 & \text { for } x>0.5\end{cases}\right.
$$


and $t_{\text {fin }}=0.1$. Finally, in test 3 , the initial data are given by

$$
\rho_{0}(x)=1, \quad u_{0}(x)=\left\{\begin{array}{ll}
-2 & \text { for } x \leqslant 0.5, \\
2 & \text { for } x>0.5,
\end{array} \quad p_{0}(x)=8\right.
$$

and $t_{\text {fin }}=0.07$. The required values $\varepsilon_{0}(x)$ are then expressed by virtue of $(3.10)$.

For all tests $1-3$, we take the simplest values $\alpha_{S}=\alpha_{P}=1$ and choose $N=400, \alpha=0.2$ and $\beta=0.1$. The main sought functions $\rho, u$ and $\varepsilon$ together with $p$ (the space graphs at time $t_{\text {fin }}$ and the corresponding space-time graphs) are presented on Figs. 1 and 2, Figs. 3 and 4, Figs. 5 and 6 for the tests 1, 2 and 3, respectively. The results on Figs. 1, 3 and 5 are completely consistent with those obtained in [8] for $N=200$.
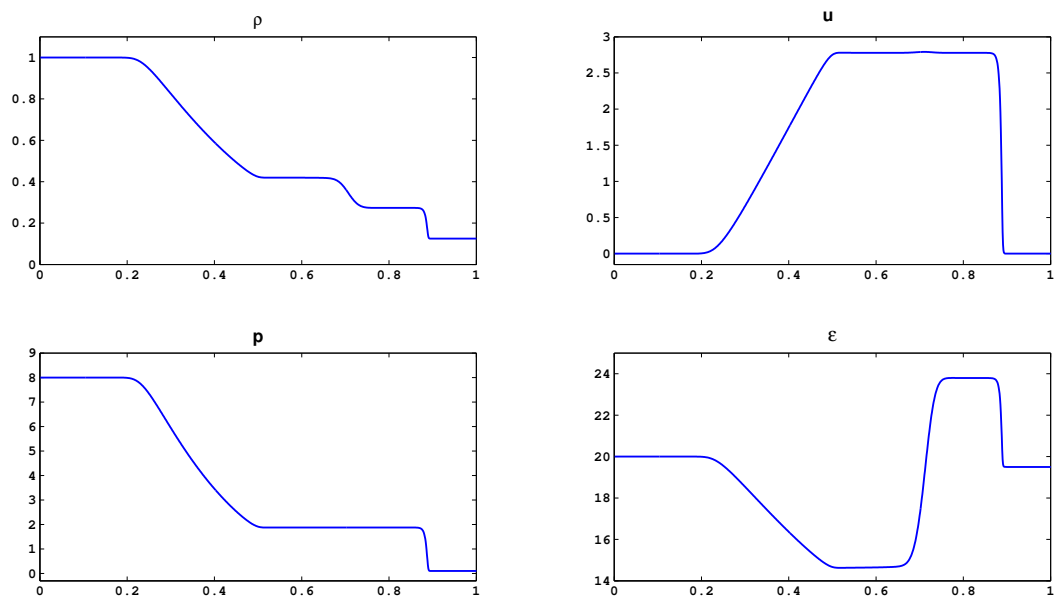

Figure 1. Test 1 (the stiffened gas): the space graphs at time $t_{\text {fin }}=0.075$.

2. The classical Van der Waals gas state equations in the form (3.7) are

$$
P(\rho, \varepsilon)=\frac{(\gamma-1) \rho(\varepsilon+a \rho)}{1-b \rho}-a \rho^{2}, \quad \Theta(\rho, \varepsilon)=\frac{\varepsilon+a \rho}{c_{v}}
$$

with $\gamma=\frac{R}{c_{v}}+1$ (for example see [6]), where $a, b, R$ and $c_{v}$ are positive physical constants. It is assumed that $0<\rho<b^{-1}$. According to formula (3.8) one can derive

$$
C_{s}^{2}=\frac{\gamma(\gamma-1)(\varepsilon+a \rho)}{(1-b \rho)^{2}}-2 a \rho
$$

Note also that

$$
p_{\rho}(\rho, \theta)=\frac{R \theta}{(1-b \rho)^{2}}-2 a \rho=\frac{(\gamma-1)(\varepsilon+a \rho)}{(1-b \rho)^{2}}-2 a \rho,
$$



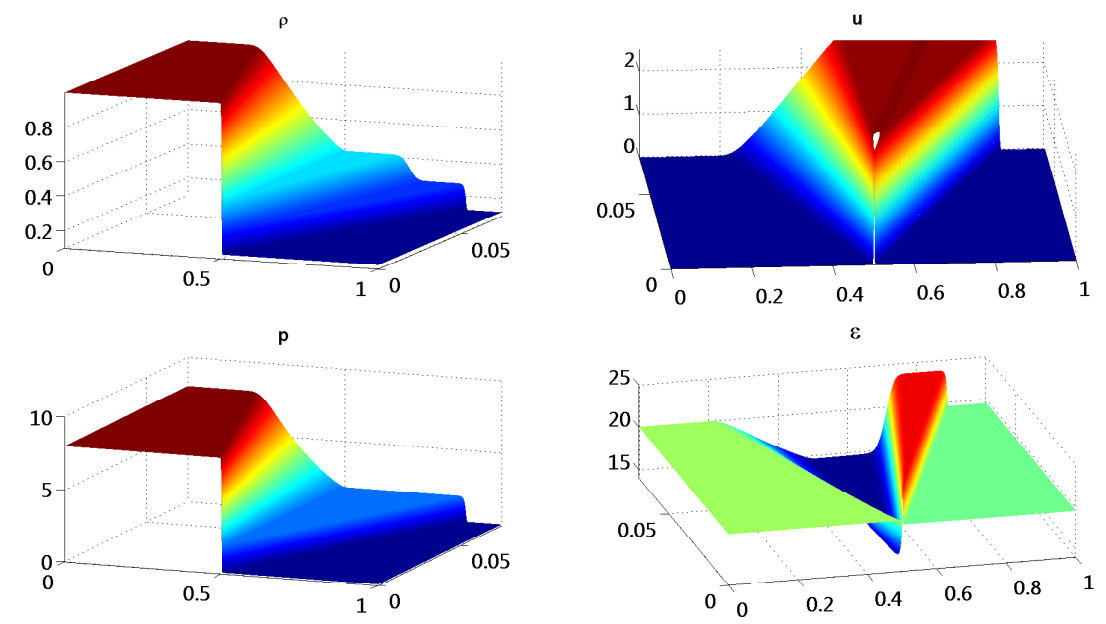

Figure 2. Test 1 (the stiffened gas): the space-time graphs.
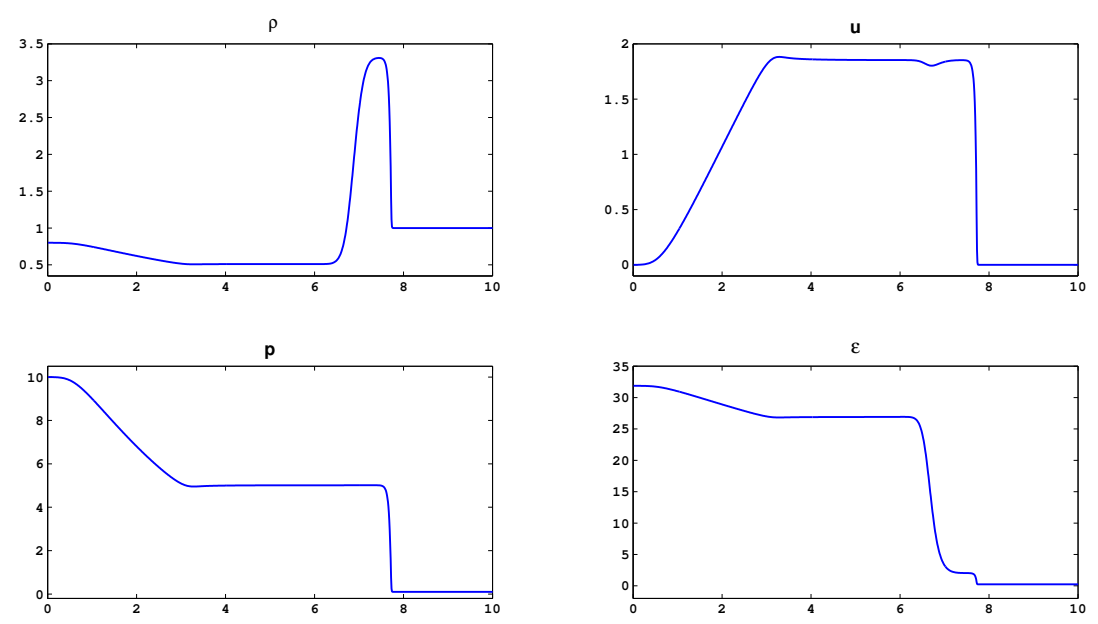

Figure 3. Test 2 (the stiffened gas): the space graphs at time $t_{\text {fin }}=0.1$. 

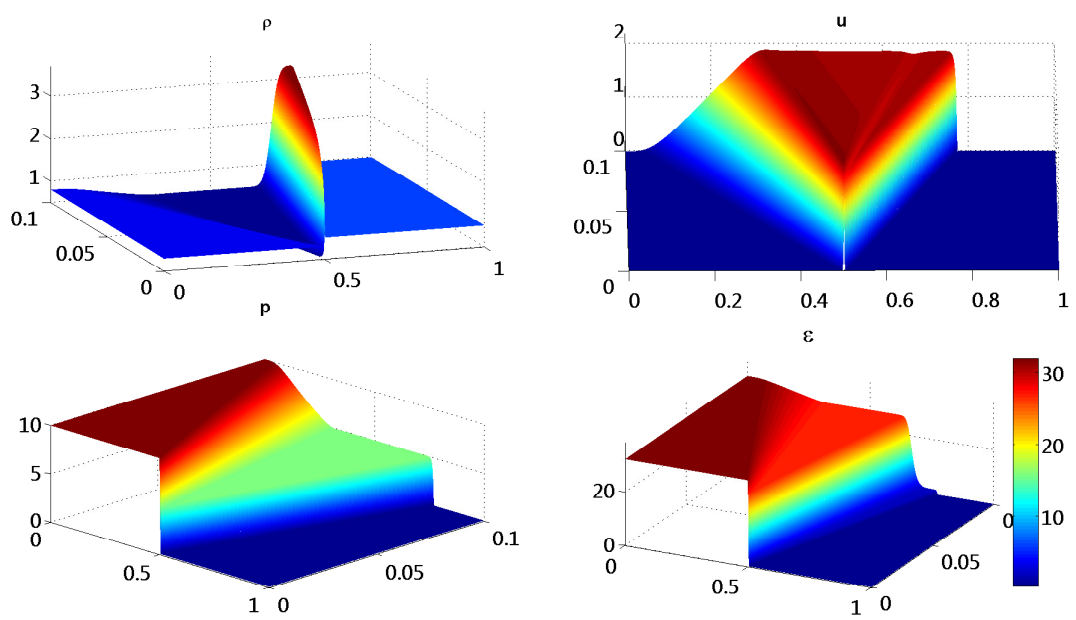

Figure 4. Test 2 (the stiffened gas): the space-time graphs.
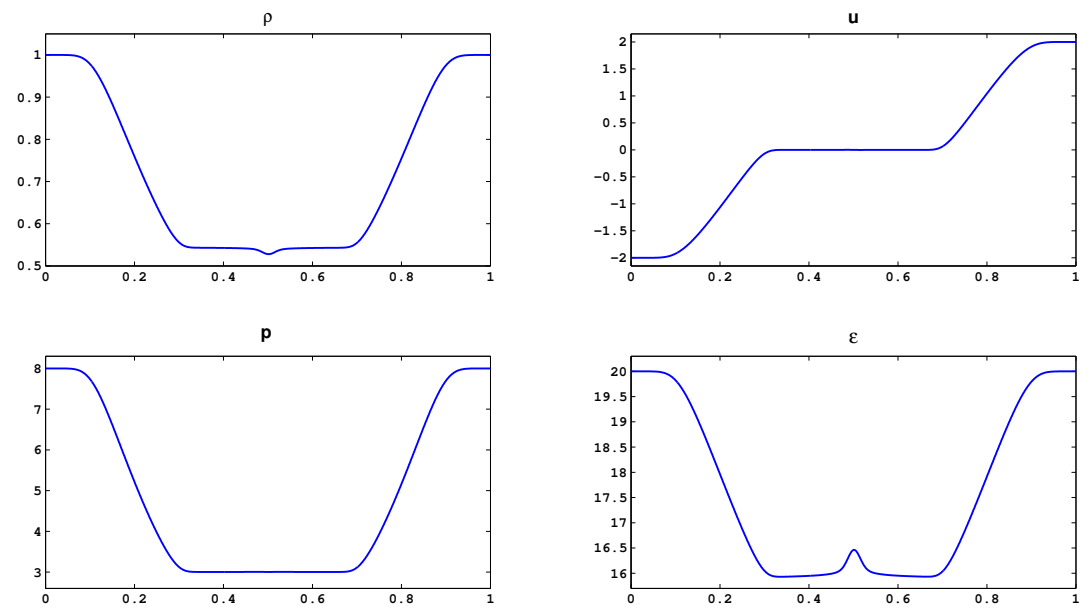

Figure 5. Test 3 (the stiffened gas): the space graphs at time $t_{\text {fin }}=0.07$. 

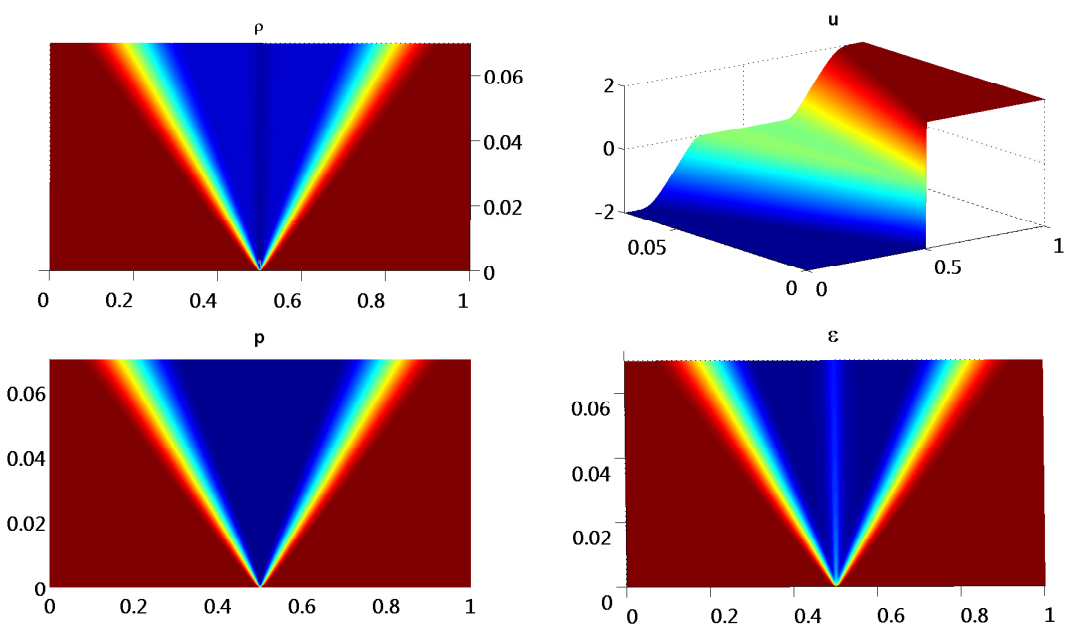

Figure 6. Test 3 (the stiffened gas): the space-time graphs.
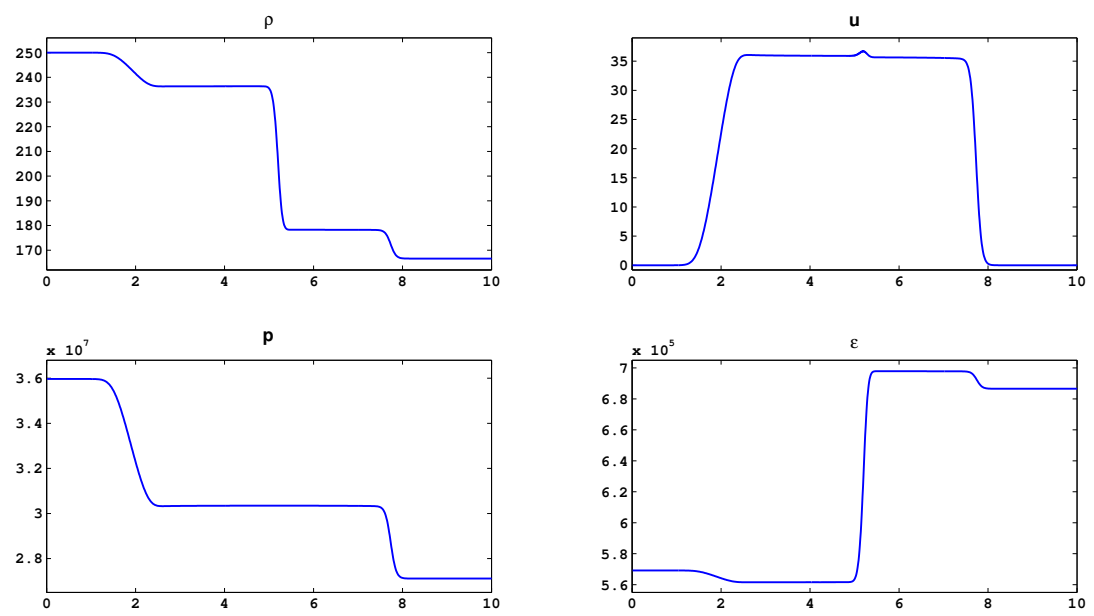

Figure 7. Test 4 (the Van der Waals gas): the space graphs at time $t_{\text {fin }}=0.005$. 

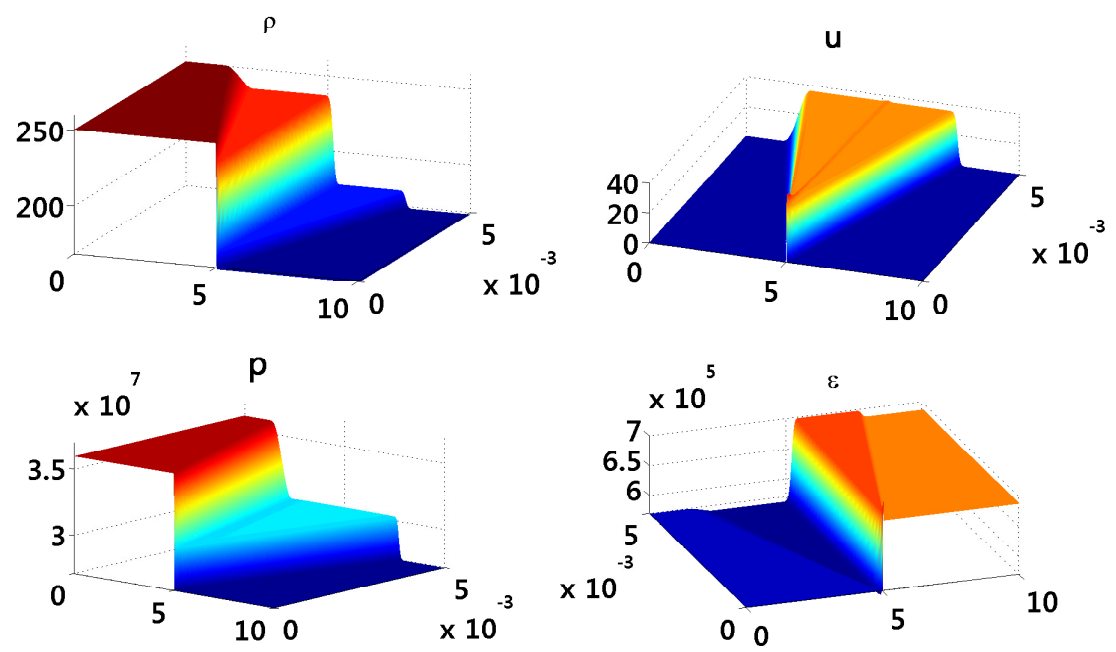

Figure 8. Test 4 (the Van der Waals gas): the space-time graphs.

and we confine ourselves to the thermodynamic stability case $p_{\rho}(\rho, \theta)>0$ (no phase change).

For the Van der Waals gas, we exploit test 5 from [1]. The chosen values of the constants are:

$$
a=1684.54, \quad b=0.001692, \quad R=461.5, \quad c_{v}=1401.88 .
$$

Also $X=10$ and the initial data are given by

$$
\rho_{0}(x)=\left\{\begin{array}{ll}
250 & \text { for } x \leqslant 5, \\
166.6 & \text { for } x>5,
\end{array} \quad u_{0}(x)=0, \quad p_{0}(x)= \begin{cases}35966778 & \text { for } x \leqslant 5 \\
27114795 & \text { for } x>5\end{cases}\right.
$$

The time $t_{\text {fin }}$ is absent in [1] so our value $t_{\text {fin }}=0.005$ is fitted to get the closest results. For this test, we once again take $\alpha_{S}=\alpha_{P}=1$ but choose now $N=700, \alpha=0.65$ and $\beta=0.1$. The functions $\rho, u$ and $\varepsilon$ together with $p$ are presented on Figs. 7 and 8 . The results on Fig. 7 are in accordance with those obtained in [1] for $N=500$.

The results of numerical experiments confirm efficiency of the constructed finite-difference scheme. Notice that a generalization of the scheme to higher dimensions is straightforward and now numerical testing of similar 2D applications is in progress.

\section{References}

[1] T. Buffard, T. Gallouet and J.-M. Herard. A sequel to a rough Godunov scheme: Application to real gases. Computers and Fluids, 29:813-847, 2000. http://dx.doi.org/10.1016/S0045-7930(99)00026-2. 
[2] B.N. Chetverushkin. Kinetic Schemes and Quasi-Gas Dynamic System of Equations. CIMNE, Barcelona, 2008.

[3] T.G. Elizarova. Quasi-Gas Dynamic Equations. Springer, Dordrecht, Heidelberg, London, New York, 2009.

[4] T.G. Elizarova and E.V. Shil'nikov. Capabilities of a quasi-gasdynamic algorithm as applied to inviscid gas flow simulation. Comput. Math. Math. Phys., 29(3):532-548, 2009. http://dx.doi.org/10.1134/S0965542509030142.

[5] F.R. Gantmacher. The Theory of Matrices. Chelsea, New York, 1971.

[6] I.A. Kvasnikov. Thermodynamics and Statistical Physics, vol. 1: Theory of Equilibrium Systems and Thermodynamics. 2nd edition. Editorial URSS, Moscow, 2002. (in Russian)

[7] Yu.V. Sheretov. Continuum Dynamics under Spatiotemporal Averaging. RKhD, Moscow-Izhevsk, 2009. (in Russian)

[8] Hua-Zhong Tang. Gas-kinetic schemes for compressible flow of real gases. Comput. Math. Appl., 41:723-734, 2001.

http://dx.doi.org/10.1016/S0898-1221(00)00316-3.

[9] Ya.B. Zeldovich and Yu.P. Raizer. Physics of Shock Waves and High-Temperature Hydrodynamic Phenomena. Academic Press, New York, 1966.

[10] A.A. Zlotnik. Classification of some modifications of the Euler system of equations. Doklady Math., 73(2):302-306, 2006.

http://dx.doi.org/10.1134/S1064562406020396.

[11] A.A. Zlotnik. Energy equalities and estimates for barotropic quasi-gasdynamic and quasi-hydrodynamic systems of equations. Comput. Math. Math. Phys., 50(2):310-321, 2010. http://dx.doi.org/10.1134/S0965542510020120.

[12] A.A. Zlotnik. Quasi-gasdynamic system of equations with general equations of state. Doklady Math., 81(2):312-316, 2010.

http://dx.doi.org/10.1134/S1064562410020419.

[13] A.A. Zlotnik. Linearized stability of equilibrium solutions to the quasigasdynamic system of equations. Doklady Math., 82(2):811-815, 2010. http://dx.doi.org/10.1134/S1064562410050352.

[14] A.A. Zlotnik. On the quasi-gasdynamic system of equations with general equations of state and a heat source. Math. Model., 22(7):53-64, 2010. (in Russian)

[15] A.A. Zlotnik and B.N. Chetverushkin. Parabolicity of the quasi-gasdynamic system of equations, its hyperbolic second-order modification, and the stability of small perturbations for them. Comput. Math. Math. Phys., 48(3):420-446, 2008. http://dx.doi.org/10.1134/S0965542508030081. 